\title{
MOLECULAR HYDROGEN EMISSION FROM PHOTODISSOCIATION REGIONS
}

\author{
Tetsuo Hasegawa \\ Institute of Astronomy, The University of Tokyo \\ 2-21-1 Osawa, Mitaka, Tokyo 181, Japan
}

\begin{abstract}
We review new observational and theoretical developments of the understanding of the $\mathrm{H}_{2}$ infrared emission in the last 5 years since the discovery of the fluorescent emission in NGC 2023. An excitation analysis of $\mathrm{H}_{2}$ in a variety of Galactic sources has revealed that in many sources the excitation is expressed as a mixture of thermal and fiuorescent components. This finding is in good agreement with theories of photodissociation regions, in which the population of $\mathrm{H}_{2}$ changes its character from pure fluorescence to thermal as the density of the region increases. The ortho/para abundance ratio of the fluorescent $\mathrm{H}_{2}$ is observed to lie within a limited range of $1.1-1.8$ which is well reproduced by depth-dependent model calculations of the ultraviolet excitation and dissociation of $\mathrm{H}_{2}$ molecules. This may be understood as due to the independent self shielding of each of the ortho- and para- $\mathrm{H}_{2}$, rather than the ortho/para abundance ratio of the predissociated $\mathrm{H}_{2}$, a low formation temperature of $\mathrm{H}_{2}$ on grains, or gas phase interchange reactions. A laser emission of molecular hydrogen discovered in the planetary nebula NGC7027 further demonstrates the nonthermal nature of the $\mathrm{H}_{2}$ emission in photodissociation regions.
\end{abstract}

\section{Introduction}

Direct observation of $\mathrm{H}_{2}$ in dense molecular clouds is very difficult and there are only two special cases in which its observation is possible : (a) thermal excitation in shocked molecular gas and (b) radiative excitation in photodissociation regions on surfaces of molecular clouds exposed to strong ultraviolet radiation.

The existence of the shocked molecular hydrogen was recognized soon after the detection of rovibrational line emission in Orion-KL (Gautier et al. 1976). Detailed studies of the level population of $\mathrm{H}_{2}$ in Orion-KL have proved the collisional excitation in shocked molecular gas (e.g., Beckwith et al. 1983; see also Brand in this volume). Measurements in other sources have shown that the population distribution is consistent with a thermal excitation within the limits of number of observed levels and observational errors, and have led people to believe that a detection of emission from vibrationally excited molecular hydrogen is a proof of shocked molecular gas.

Detection of fluorescent $\mathrm{H}_{2}$ emission in the reflection nebula NGC 2023 and other sources changed this situation dramatically (Gatley et al. 1987; Hayashi et al. 1985; Sellgren 1986). A detection of an $\mathrm{H}_{2}$ emission line (e.g., $v=1-0 S(1)$ at $\lambda 2.12 \mu \mathrm{m}$ ) does not by itself prove an existence of a shock and any conclusion on the nature of the excitation of $\mathrm{H}_{2}$ molecules is difficult before a detailed analysis of the population of levels with $v \geq 2$ is made.

In this paper we review recent observational and theoretical development of the understanding of the $\mathrm{H}_{2}$ emission in the last 5 years since the discovery of the infrared fluorescence. 


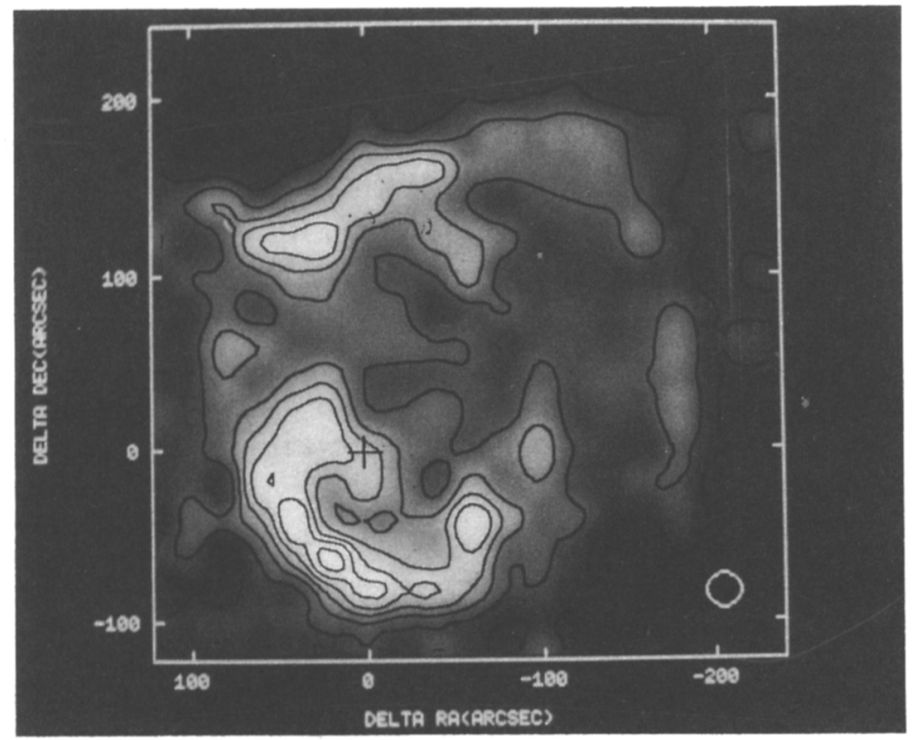

FIG. 1. - A map of the emisssion in the $v=1-0, S(1)$ line of $\mathrm{H}_{2}$ from the reflection nebula NGC 2023. The position of the exciting star HD 37903 is indicated by a cross. (from Gatley et al. 1987.)

\section{Fluorescent Molecular Hydrogen}

Figure 1 shows the distribution of the $\mathrm{H}_{2} v=1-0 S(1)$ emission in NGC 2023. This reflection nebula is situated on the surface of a dark cloud facing toward us and is illuminated by a B1.5 V star. The molecular gas on the surface of the cloud is exposed to soft (i.e., non-ionizing) ultraviolet radiation from the star and a photodissociation region (Tielens and Hollenbach 1985) is formed.

In this region, $\mathrm{H}_{2}$ molecules absorb ultraviolet photons in the Lyman and Werner bands at $912-1108$ A to become electronically excited. The excited $\mathrm{H}_{2}$ molecules immediately emit ultraviolet photons to return to the ground electronic state. Ten percent of this transition leads to dissociation of $\mathrm{H}_{2}$ molecules while the rest results in $\mathrm{H}_{2}$ molecules in the ground electronic state with vibrational excitation, which cascade down via rovibrational transitions, i.e., fluorescence.

The detailed level population of the fluorescent molecular hydrogen first measured in NGC 2023 is shown in Figure 2 in comparison with that in Orion-KL, a prototypical shocked source. Comparison of the two panels in Figure 2 readily shows three remarkable points as follows (Hasegawa et al. 1987):

1. NGC 2023 shows two separate sequences of energy levels corresponding to the para (even $J$ ) and ortho (odd $J$ ) forms of $\mathrm{H}_{2}$. As the statistical weight, $g_{u}$, applied in the figure includes the spin degeneracies, the para and ortho levels should align on a single sequence if the ortho/para abundance ratio is 3 , as is indeed the case for OrionKL shown in the lower panel. The separation between the two sequences in the $v=1$ and $v=2$ states corresponds to the ortho/para ratio of 2.0 and 1.4, respectively.

2. Each of the two sequences of the level population in NGC 2023 is characterized by a high vibrational excitation temperature, $T_{v}$, and a low rotational excitation tem- 

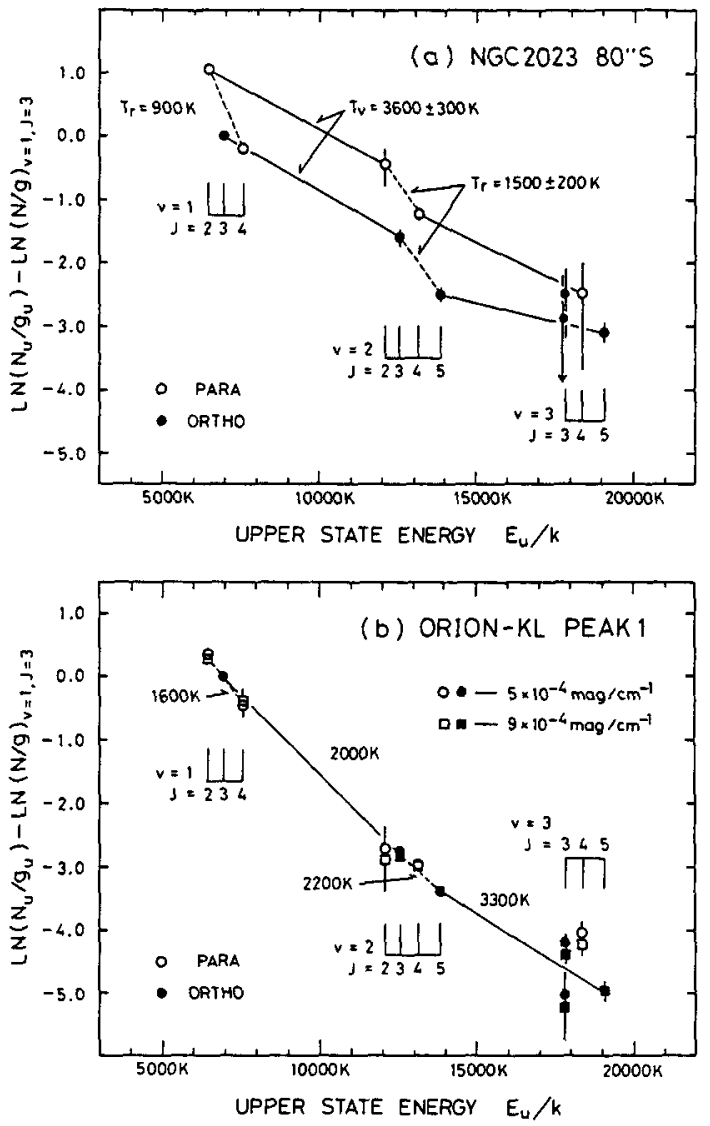

FIG. 2. - The relative level population of $\mathrm{H}_{2}$ in NGC2023 (a) and Orion-KL (b). For Orion-KL, reddening correction has been applied based on the two estimates indicated in the upper right-hand corner. Excitation temperatures measured from the slopes of the lines are shown. (from Hasegawa et al. 1987.)

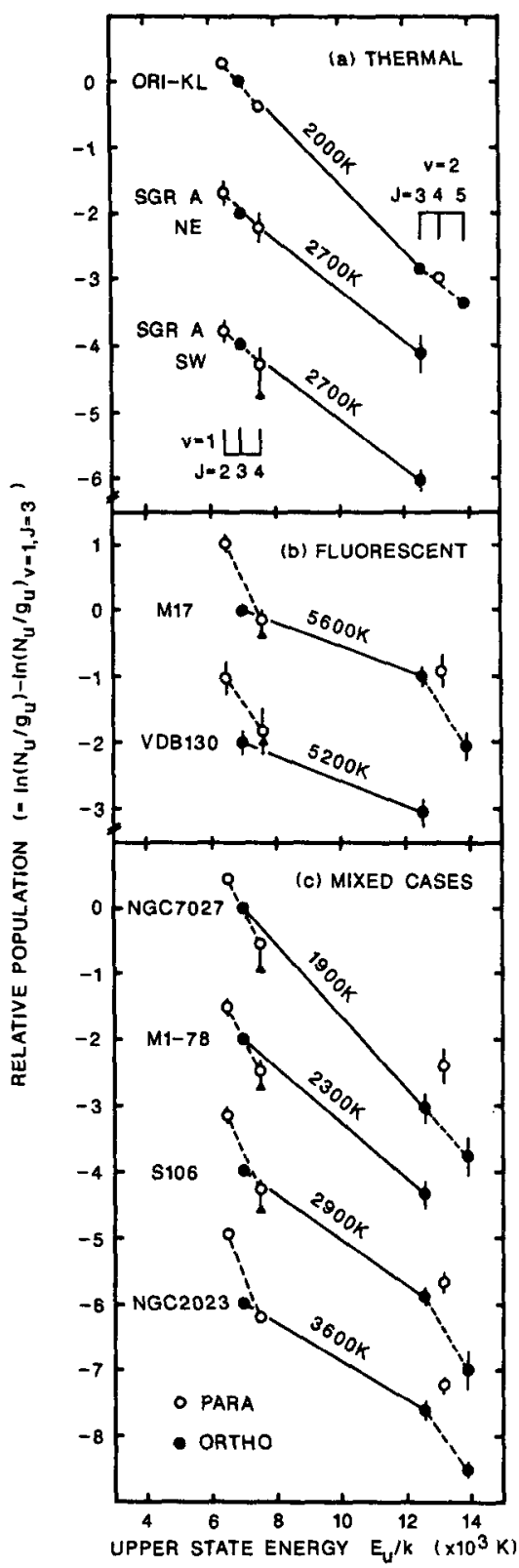

FIG. 3. - The relative level population of $\mathrm{H}_{2}$ in a variety of Galactic objects. The sources are grouped according to the three phenomenological categories of the excitation; see Section 3 in the text. (from Tanaka et al. 1989.) 
perature, $T_{r}$. This is in contrast to the case of Orion-KL, in which the levels at higher energy are characterized by higher excitation temperatures without any systematic difference between the vibrational and rotational temperatures.

3. The two sequences of population in NGC 2023 show similar excitation characteristics. Both the populations of para- $\mathrm{H}_{2}$ and ortho- $\mathrm{H}_{2}$ are consistent with the same $v=2-1$ vibrational temperatures and the same rotational temperatures in the $v=2$ state. This suggests that the ortho/para ratio measured from the separation of the two sequences may represent the ratio of the total abundance of para- $\mathrm{H}_{2}$ and ortho- $\mathrm{H}_{2}$.

The high vibrational temperature and the low rotational temperature found in NGC 2023 (point [1] above) are in good agreement with theoretical expectations by Black and Dalgarno (1976), Takayanagi, Sakimoto, and Onda (1987), and Black and van Dischoek (1987).

\section{A Survey}

A survey of fluorescent molecular hydrogen in a variety of Galactic sources were made by Tanaka et al. (1989). The objects included the HII regions M17 and S106, the reflection nebula vdB 130 , the planetary nebula NGC 7027, the Galactic center, and the enigmatic object M1-78 which is thought to be a distant HII region. The results are shown in the population diagrams in Figure 3.

The excitation of molecular hydrogen is classified into three phenomenological categories:

Thermal Sources - Sources in this category have population distribution which align along a smooth line or curve without significant differences between the vibrational and rotational temperatures.

Fluorescent Sources - Sources in this category show population distribution in good agreement with that theoretically expected for fluorescence in low density ( $n_{\text {total }}$ $\lesssim 10^{4} \mathrm{~cm}^{-3}$ ) photodissociation regions. The vibrational temperature is as high as $\gtrsim 5000$ $\mathrm{K}$ while the rotational temperature is only about $1000 \mathrm{~K}$. A marked departure of the ortho/para ratio from 3 is observed.

Mixed Cases - This group of sources exhibit population distribution which is in between the two extreme cases above and can be expressed as a mixture of the two excitation mechanisms. The ortho/para ratio observed in the $v=1$ state is genarally larger than that in the $v=2$ state. This reflects the more pronounced contribution from thermal emission in the $v=1$ state. The prototypical fluorescent source NGC 2023 falls in this category together with the HII regions S106 and M1-78. Surprizingly, the planetary nebula NGC 7027, which has been widely accepted as a source of shocked $\mathrm{H}_{2}$, showed a sign of fluorescent contribution; it has even a maser action in a $v=3-2$ transition probably pumped by UV radiation (see Section 5).

To assess the relative importance of the thermal and fluorescent excitation in the mixed cases, Tanaka et al. (1989) developed a decomposition algorithm which uses the theoretical population of low density fluorescence (Takayanagi, Sakimoto, and Onda 1987; Black and van Dischoek 1987) as a template for the purely fluorescent component. The observed ortho/para ratio in the mixed cases becomes larger and approaches 3, the equilibrium value at high temperature, as the contribution of the thermal emission increases. After separation of the thermal contributionfrom the observed spectra, the real ortho/para ratio of the fluorescent $\mathrm{H}_{2}$ can be estimated. 


\section{TABLE 1}

DeCOMPOSITION OF THERMAL AND FLUORESCENT EMISSION

\begin{tabular}{|c|c|c|c|c|}
\hline \multirow[b]{2}{*}{ SOURCE } & \multicolumn{2}{|c|}{$\begin{array}{c}\text { THERMAL } \\
\text { FrACTION }^{\mathbf{a}} T_{\text {ex }}^{\mathrm{b}}\end{array}$} & \multicolumn{2}{|c|}{$\begin{array}{c}\text { Radiative } \\
\text { ORtho/Para Ratio }\end{array}$} \\
\hline & $\%$ & $\mathbf{K}$ & $v=1$ & $v=2$ \\
\hline Orion-KL & 100 & 2000 & . & \\
\hline Sgr A NE. & $>90$ & 2500 & . & \\
\hline Sgr A SW .... & $>90$ & 2500 & . & $\ldots$ \\
\hline NGC $7027 \ldots$. & 90 & 1200 & . & $1.0(0.3)$ \\
\hline M1-78 ............... & 80 & 1200 & $\ldots$ & $\ldots$ \\
\hline \$106 $\ldots \ldots \ldots \ldots \ldots \ldots \ldots$ & 65 & $<1400$ & $1.4(0.2)$ & $1.4(0.3)$ \\
\hline NGC $2023 \ldots \ldots \ldots \ldots$ & 60 & 2000 & $1.1(0.2)$ & $1.2(0.2)$ \\
\hline $\mathrm{M} 17 \quad \ldots \ldots \ldots \ldots \ldots \ldots$ & $<10$ & $\ldots$ & $1.8(0.3)$ & $1.7(0.3)$ \\
\hline vdB $130 \ldots \ldots \ldots \ldots$ & $<15$ & $\cdots$ & $1.7(0.4)$ & $\ldots$ \\
\hline
\end{tabular}

- Fraction of thermal component in $v=1 \multimap S(1)$ line emission. Typical errors are $10 \%$.

b The excitation temperature for the thermal component. Typical errors are $200 \mathrm{~K}$.

c Numbers in parentheses are uncertainties which arise mainly from observational errors.

The results of decomposition are shown in Table 1. The ortho/para ratio of the fluorescent $\mathrm{H}_{2}$ in the present rather diverse sample is found to lie within the limited range of $1.1-1.8$ (we exclude NCG 7027 for now; see Section 5). The near constancy of the ortho/para ratio is rather striking, because a large range of values (including $\sim 0$ ) would be anticipated if the fluorescent ortho/para ratio corresponds to that in the molecular cloud before radiative excitation. The results in Table 1 may, rather, suggest that the ortho/para ratio of the fluorescent $\mathrm{H}_{2}$ is mainly determined by elemental processes of fluorescence. Further consideration of this point wil be given in the next section.

The thermal emission separated in the decomposition described above does not necessarily originate from shocked molecular gas. Models of dense photodissociation regions have shown that when the density is as high as $\gtrsim 10^{5} \mathrm{~cm}^{-3}$ the gas temperature rises up to $\sim 10^{3} \mathrm{~K}$ and that at that high temperature the collisional excitation/deexcitation dominates the ultraviolet pumping to thermalize the level population at the gas temperature (Hollenbach 1988; Sternberg and Dalgarno 1989; Burton, Hollenbach, and Tielens 1989). The thermal component found in some of the sources may be due to collisional excitation in hot, dense photodissociation regions.

\section{The Significance of the Observed Ortho/Para Ratio}

The universality of the ortho/para ratio found for the fluorescent $\mathrm{H}_{2}$ is rather striking. Hasegawa et al. (1987) and Takayanagi, Sakimoto, and Onda (1987) tried to attribute the observed low ortho/para ratio in NGC 2023 to the process of $\mathbf{H}_{2}$ formation. In the fluorescent zone, photodissociation of $\mathrm{H}_{2}$ molecules is balanced approximately by reformation of $\mathrm{H}_{2}$ on dust grains. If nascent $\mathrm{H}_{2}$ molecules have a rovibrational population in a Boltzmann distribution at a formation temperature, $T_{f}$, which is low compared 
with the energy difference between the $v=0, J=0$ and $J=1$ levels $(\Delta E / k=170.5$ $\mathrm{K})$, para $(J=0) \mathrm{H}_{2}$ is preferentially formed to make the ortho/para ratio considerably smaller than 3. A model incorporating the ultraviolet excitation/dissociation, the $\mathrm{H}_{2}$ formation on grains, and the exchange reaction between $\mathrm{H}_{2}$ and $\mathrm{H}$ was constructed by Takayanagi, Sakimoto, and Onda (1987) which could reproduce the observed data with $T_{f}=60-70 \mathrm{~K}$. This model, however, did not take the dependence of the ultraviolet excitation on the depth from the cloud surface into account and used a single value for the excitation rate via absorption in each of the Lyman and Werner lines.

Black and van Dischoek (1987) performed a depth-dependent calculation for the abundance and the excitation of $\mathrm{H}_{2}$ for a series of model photodissociation regions. They noted that (1) the exchange reaction between $\mathrm{H}_{2}$ and $\mathrm{H}^{+}$is not negligible in the outermost layer of a cloud where photoionization of vibrationally excited $(v \geq 4) \mathrm{H}_{2}$ supplies ample $\mathrm{H}^{+}$, and that (2) the excitation rate is very level-specific at any particular depth to a cloud. They claimed that the low formation temperature is not required in order to reproduce the low ortho/para ratio.

Closer examination (from observer's point of view) of the models of Black and van Dischoek (1987) reveales a remarkable feature. The ortho/para ratio estimated from the intensities of the $v=2-1, S(1), S(2)$, and $S(3)$ lines predicted by their calculation falls within a limited range $1.5-1.8$ without any strong dependence on the density $\left(n_{H}=10^{2}-10^{3.5} \mathrm{~cm}^{-1}\right)$ and the intensity of the ultraviolet radiation $\left(1-10^{4}\right.$ times the interstellar radiation field) for their standard choice of gas temperature ( $T=100$ $\mathrm{K})$, grain properties, and $\mathrm{H}_{2}$ formation model. Its dependence on the gas temperature is also weak; at $n_{T}=10^{3.5} \mathrm{~cm}^{-3}$, the ortho/para ratio changes from 1.4 to 2.0 for a large temperature rise from $30 \mathrm{~K}$ to $300 \mathrm{~K}$. Indeed the model calculation by Sternberg and Dalgarno (1989) which incorporates the depth dependence of the gas temperature determined by the local thermal balance gives a ratio of 1.6 for $n_{T}=10^{3} \mathrm{~cm}^{-3}$ and the ultraviolet field $10^{3}$ times the interstellar field, in good agreement with the ratios found in Black and van Dishoek models. This means that the interchange reactions $\left(\mathrm{H}_{2}+\mathrm{H}\right.$ and $\mathrm{H}_{2}+\mathrm{H}^{+}$) do not show a major contribution in determining the ortho/para ratio of the fluorescent $\mathrm{H}_{2}$ in this density range.

The constancy of the ortho/para ratio apparent in depth-dependent models may be naturally understood if we remember that the ortho and para molecular hydrogen act as two different species with different sets of electronic transitions which absorb ultraviolet photons. The sharp transition from predominantly atomic to predominantly molecular forms of hydrogen in photodissociation regions is due to self-shielding in the ultraviolet absorption lines; the dissociation rate drops suddenly as soon as the strong absorption lines becomes optically thick (see, e.g., Figure 1 in Black and van Dischoek 1987). In other words the photodissociation stops at a depth where the ultraviolet photons in the strong absorption lines are exhausted. This situation occurs for each of ortho and para molecular hydrogen independently if overlaps of ultraviolet lines are neglected. The change in the ortho/para abundance ratio causes a slight shift of the relative position of the dissociation fronts of ortho- and para- $\mathrm{H}_{2}$ with the ratio of the number of absorbed ultraviolet photons unchanged. As the column density of vibrationally excited ortho- or para- $\mathrm{H}_{2}$ in a less dense $\left(n_{H} \lesssim 10^{4} \mathrm{~cm}^{-3}\right)$ photodissociation region is roughly proportional to the ultraviolet photons absorbed, the observed ortho/para ratio of the fluorescent $\mathrm{H}_{2}$ is determined primarily by elementary processes, i.e., the oscillator strengths and wave- 
lengths of Lymann and Werner absorption lines and the relative poulation of the lower levels of these lines which is determined by Einstein coefficients of rovibrational transitions. The universality of the observed orth/para ratio of the fluorescent component and its constancy reproduced in depth-dependent model calculations may be understood in this way.

\section{Infrared Laser Emission of $\mathrm{H}_{2}$ in NGC $\mathbf{7 0 2 7}$}

The nonthermal nature of the excitation of molecular hydrogen in photodissociation regions becomes most prominent in the planetary nebula NGC 7027. In 1989, Hasegawa, Tanaka, and Brand (1989) discovered an extraordinarily strong emission of the $\mathrm{H}_{2}$ $v=3-2, S(2)$ emission at $\lambda 2.2864 \mu \mathrm{m}$ in NGC 7027. This line was originally found in the Fourier spectrum taken by Treffers et al. (1976) and remained unidentified since then. The population distribution of $\mathrm{H}_{2}$ analyzed by Hasegawa, Tanaka, and Brand exhibuts highly nonthermal nature with $v=3, J=4,5$, and 6 levels and $v=2, J=4$ level significantly overpopulated. The $v=3-2, S(2)$ transition is inverted, and the contribution of induced emission, $B I_{\nu} / c$, is comparable to that of spontaneous emission, $A$, where $A$ and $B$ are Einstein coefficients. These are the characteristics of Laser emission.

Detection of the unidentified line at $\lambda 2.2864 \mu \mathrm{m}$ in other sources are found in the literature. Isaacman (1984) reports detection of this line in two planetary nebulae IC 5117 and NGC 6572. In addition, Thompson, Lebofsky, and Rieke (1978) reports detection of this line in the nuclear region of the Seyfert galaxy NGC 1068. Although measurements at higher spectral resolution are required to assign these reported features observed at relatively low resolution to the $v=3-2, S(2)$ line of molecular hydrogen, these may suggest that the laser emission of this line is a relatively common phenomenon.

\section{Conclusions}

The progress of the understanding of the $\mathrm{H}_{2}$ emission in general and especially in photodissociation regions has been huge as reviewed in this paper, and explosive increase of observational information is still going on with continuous innovation of observational capabilities in infrared astronomy. In the near future, studies of the $\mathrm{H}_{2}$ emission in external galaxies will be made in the detail we can attain now for Galactic objects, and for the Galactic objects we will be able to visualize the detailed structure of the photodissiciation regions which we can only observe in theoretical models. And beynd these is a long-standing target, i.e., detection of the $v=0-0, J=0-2$ and $1-3$ lines from the bulk of a dense molecular cloud, which should open the door to an observational approach to the formation mechanism of molecular hydrogen.

Ackowledgements We wish to thank our collaborators I. Gatley, M. Tanaka, M. Hayashi, S. Hayashi, N. Kaifu, P. Brand, and R. Garden. Illuminating discussion with K. Takayanagi, $K$. Sakimoto, and $K$. Onda and inspiring comments received at the conference from $A$. Dalgrno and $\mathrm{P}$. Solomon were very valuable in finishing this paper in its final form.

\section{References}

Beckwith, S., Evans, N, Gatley, I., Gull, G., and Russel, R. 1983, Ap. J., 264, 152.

Black, J., and Dalgarno, A. 1976, Ap. J., 203, 132.

Black, J., and van Dischoek, E. 1987, Ap. J., 322, 412.

Gatley et al. 1987, Ap. J. (Letters), 318, L73. 
Gautier, T., Fink, U., Treffers, R., and Larson, R. 1976, Ap. J. (Letters), 207, L129. Hasegawa, T., Gatley, I., Garden, R., Brand, P., Ohishi, M., Hayashi, M., and Kaifu, N. 1987, Ap. J. (Letters), 318, L77.

Hasegawa, T., Tanaka, M., and Brand, P., 1989, Ap. J. (Letters), submitted.

Hayashi, M., Hasegawa, T., Gatley, I., Garden, R., and Kaifu, N 1985, M. N. R. A. S., 215, 31P.

Hollenbach, D. 1988, Astr. Lett. and Comm., 26, 191.

Isaacman, R. 1984, Astr. Ap., 130, 151.

Sellgren, K. 1986, Ap. J., 305, 399.

Sternberg, A. and Dalgarno, A. 1989, Ap. J., 338, 197.

Takayanagi, K., Sakimoto, K., and Onda, K. 1987, Ap. J. (Letters), 318, L81.

Tanaka, M., Hasegawa, T., Hayashi, S., Brand, P., and Gatley, I. 1989, Ap. J., 336, 207.

Thompson, R., Lebofsky, M., and Rieke, G. 1978, Ap. J. (Letters), 222, L49.

Tielens, A., and Hollenbach, D. 1985, Ap. J., 291, 722.

Treffers, R., Fink, U., Larson, H., and Gautier, T. 1976, Ap. J., 209, 793. 